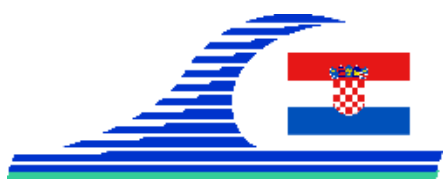

\title{
Etude physique et environnementale des sédiments du port de commerce de Sfax
}

Conférence Méditerranéenne Côtière et Maritime

EDITION 4, SPLIT, CROATIA (2017)

Coastal and Maritime Mediterranean Conference

Disponible en ligne - http://www.paralia.fr - Available online

Abdelwaheb BEN SLAMA ${ }^{1}$, Nesma FEKI ${ }^{1}$, Anis KAMOUN ${ }^{2}$

1. Unité de recherche géotechnique, environnementale et matériaux civils, Route de Soukra, BP 3000 Sfax, Tunisie.

benslama_abdelwaheb@yahoo.fr,nissma.feki@gmail.com

2. Office de la marine marchande et des ports OMMP

a.kamoun@ommp.nat.tn

\section{Résumé :}

Avec l'envasement des fonds portuaires, le dragage devient une composante essentielle des activités portuaires. Pour maintenir ou restituer l'accès de ces infrastructures aux bateaux d'assez fort tonnage, il s'avère indispensable de procéder régulièrement à des dragages d'entretien. Les ports de Sfax (port de commerce et port de pêche) considérés parmi les ports les plus importants de la Tunisie, font l'objet d'opérations de dragage tous les dix ans en moyenne. Face aux quantités importantes de sédiments dragués (1.05 $\mathrm{Mm}^{3}$, la solution classique adoptée était le relargage en mer. Mais les mentalités ont changé et le respect de l'environnement prend une place de plus en plus importante dans les processus décisionnels. La valorisation est la solution la plus écologique pour la gestion de ces sédiments qui ont un impact sur le milieu aquatique. Par ailleurs dans un contexte d'épuisement des ressources naturelles, ces sédiments représentent une source potentielle importante de matériaux alternatifs pour des usages en génie civil (matériaux routiers, matériaux cimentaires....). Une telle valorisation nécessite une caractérisation physico-chimique, minéralogique et environnementale de ces sédiments afin d'avoir une connaissance approfondie de ces propriétés et évaluer ainsi leur degré de pollution afin de prévenir tout risque sur l'environnement.

Dans ce cadre, une première étude a été réalisée sur la caractérisation des sédiments du port de pêche de Sfax (FEKI \& MBARKA, 2015). Dans ce papier sont présentés les principaux résultats de l'étude de caractérisation physique et environnementale des sédiments du port de commerce de Sfax. La détermination de ces propriétés physiques (granulométrie, limites d'Atterberg, teneur en COT) permet de classer ces sédiments selon le GTR (2000) en vue d'une valorisation dans des applications routières. Un grand intérêt est attribué à l'aspect environnemental et à l'attribution de la source de pollution de ces sédiments.

Mots-clés : Sédiments marins, Port de commerce de Sfax, Propriétés physicochimiques, caractérisation minéralogique et environnementale. 
Mediterranean rocky coasts:

Features, processes, evolution and problems

\section{Introduction}

La politique nationale Tunisienne du secteur de transport maritime et des ports vise l'amélioration constante de l'efficacité et de la qualité des services, ainsi que le renforcement de la sécurité maritime tout en assurant la conservation de l'environnement marin. La Tunisie dispose de six ports principaux : Bizerte, RadèsGoulette, Sousse, Sfax, Gabès et Zarzis. Le port de commerce de Sfax occupe la troisième position sur le plan d'échange de marchandises.

Le dragage des sédiments décantés dans les bassins des ports est indispensable pour assurer le bon trafic intérieur. Le dragage se fait en moyenne tous les dix ans et peut atteindre 8.5 Millions de mètres cubes $\left(\mathrm{Mm}^{3}\right)$ pour l'ensemble des ports de la Tunisie. Le dernier dragage effectué dans le port de commerce de Sfax date de 2009 et la quantité de sédiments dragués est estimée de $1.05 \mathrm{Mm}^{3}$.

Ces sédiments ont fait l'objet d'une étude environnementale par deux bureaux d'étude, BCEOM/SCET-Tunisie (OMMP, 2007). Les résultats de ces études ont montré de fortes concentrations en Phosphore, Cadmium, Cuivre, Zinc et Hydrocarbures et qui dépassent par endroit les seuils d'acceptabilité des rejets en mer. La solution de gestion de ces sédiments était le clapage en mer à quelques kilomètres de la ville de Sfax. Le dépôt est effectué en masse pour éviter la dispersion des sédiments et en étage par ordre décroissant en teneurs polluants. Le tout est couvert par une couche de sable propre sur une épaisseur d'une vingtaine de centimètres. Le dépôt est suivi dans le temps pour s'assurer de la stabilité des talus.

Dans le cadre d'une meilleure gestion de ces sédiments on vise la caractérisation des sédiments des ports de Sfax. Après la caractérisation des sédiments du port de pêche de Sfax (FEKI \& MBARKA, 2015), le présent travail s'intéresse à l'étude des sédiments du port de commerce de Sfax. Dans la première partie de l'étude sont donnés les paramètres physiques et les concentrations des principaux éléments polluants des sédiments échantillonnés dans le port. La deuxième partie est consacrée à la caractérisation minéralogique des sédiments. Cette étude permet de classer ces sédiments en tant que matériau routier et de prévoir une solution de valorisation dans le domaine civil.

\section{Environnement du port de commerce de Sfax et localisation des échantillons}

Le port de commerce de Sfax se trouve sur la côte est de la ville de Sfax, il occupe une superficie de 48.8 Ha. Son architecture est présentée sur la figure 1. Il se compose de trois bassins principaux : bassin principal (BL), bassin NPK (BN) et bassin des voiliers (BV), une zone de liaison BL et un chenal d'accès marine au port qui s'étend sur $4.7 \mathrm{~km}$ de long et $60 \mathrm{~m}$ de largeur en dehors du port.

Les ports de Sfax (port de commerce et port de pêche) sont construits en proximité de l'ancienne entreprise de production de l'acide phosphorique (NPK) du côté Nord. Du 
Mediterranean rocky coasts:

côté sud, ils sont limités par la saline de Sfax qui elle-même avoisine la société de production des engrais phosphatés et de l'acide phosphorique (SIAPE).

Une étude de dépollution et de réhabilitation des côtes sud de Sfax réalisée en 2008 (COMETE ENGINEERING, 2008) a montré que la propagation des métaux lourds notamment le Cadmium, s'étend depuis la SIAPE jusqu'au port de commerce (figure 2). L’hydrodynamisme marin le long de la côte a contribué à la dispersion de ces éléments polluants dans les sédiments marins sur une surface de 100 hectares. Les concentrations les plus élevées sont enregistrées dans les sédiments du port de commerce. Cette amplification s'explique par la chute des phosphates dans les bassins du port au cours des opérations d'exportation.

Une campagne d'échantillonnage a été réalisée du 22 janvier au 22 avril 2016. La localisation des 20 d'échantillons sont présentés sur la figure 1.
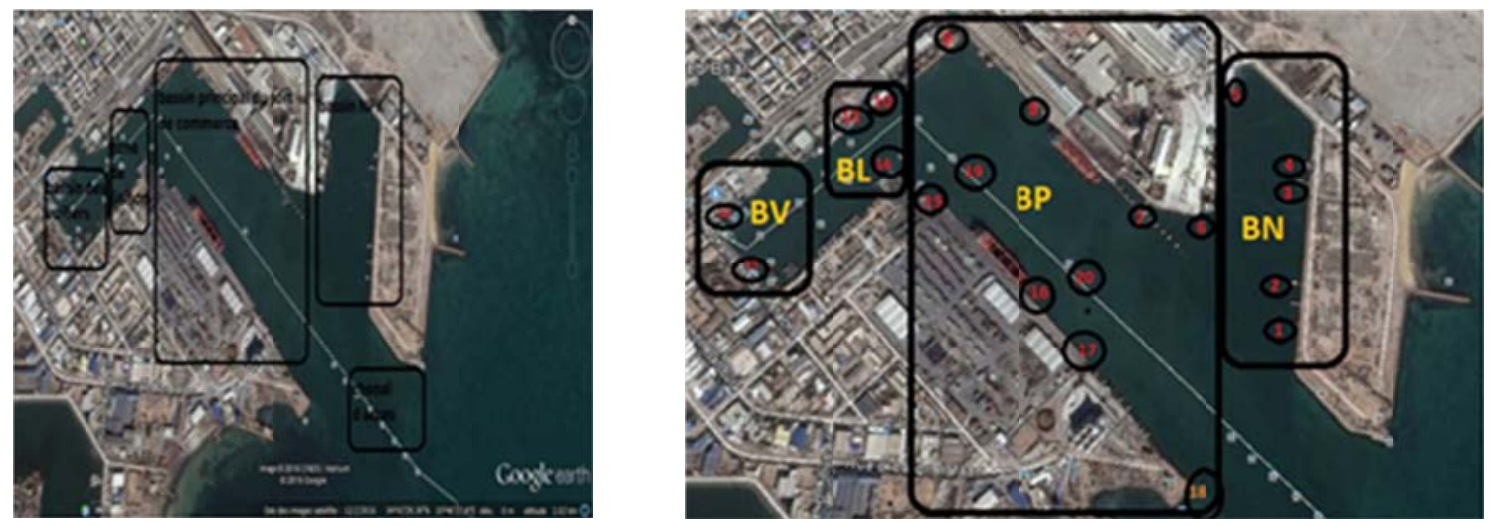

Figure 1. Localisation des prélèvements de sédiments dans les bassins du port de Sfax.

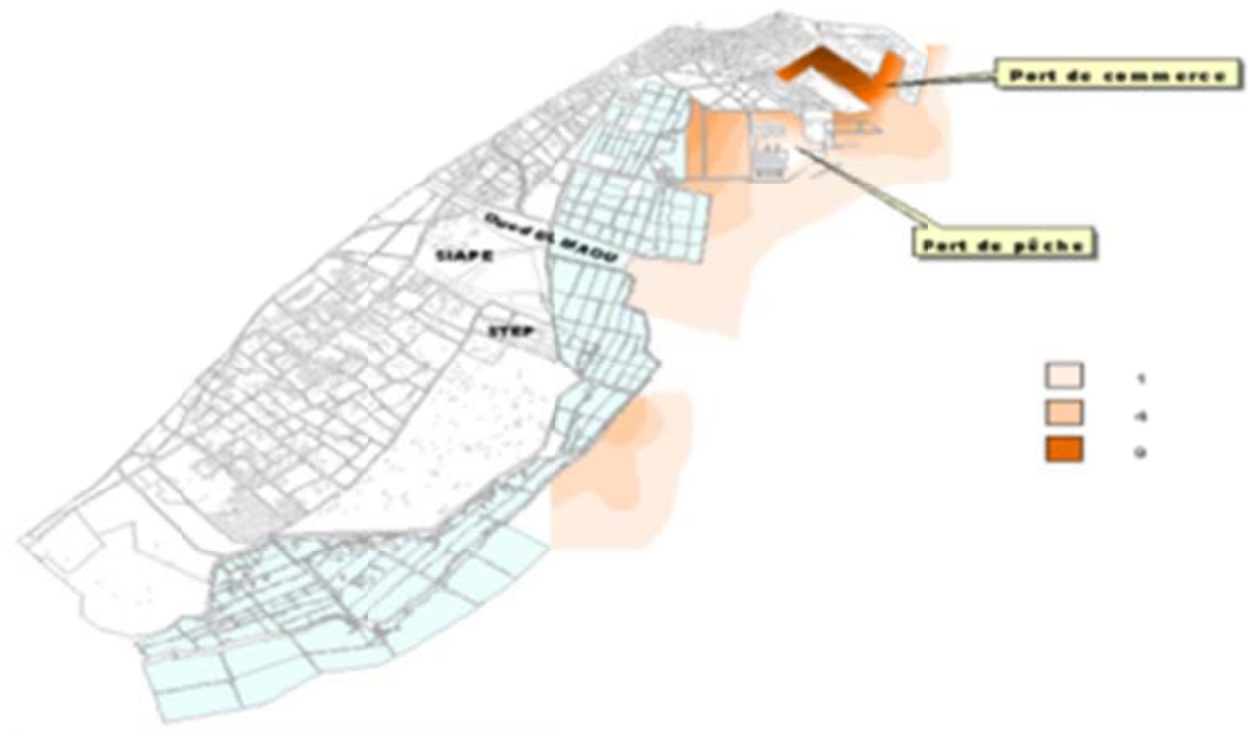

Figure 2. Répartition du cadmium dans les sédiments marins $(\mathrm{mg} / \mathrm{kg})$, (COMETE ENGINEERING, 2008). 
Mediterranean rocky coasts:

Features, processes, evolution and problems

\section{Caractérisation des sédiments du port de commerce de Sfax}

\subsection{Caractérisation physique}

L'observation macroscopique des échantillons montre une couleur grisâtre, des débris de coquilles, des indices d'hydrocarbures et des fortes teneurs en eau. La caractérisation physico-chimique concerne la distribution granulométrique, la teneur en eau, la teneur en carbone organique, la teneur en carbonate de calcium et les concentrations en métaux lourds. L'ensemble de résultats sont résumés sur le tableau1.

Les résultats des différents essais de la caractérisation physique des sédiments sont exprimés sous forme des valeurs moyennes des 20 échantillons.

Tableau 1. Caractéristiques physiques des sédiments du port de commerce de Sfax.

\begin{tabular}{|c|c|c|c|c|}
\hline \multicolumn{5}{|l|}{ Caractéristiques physiques } \\
\hline Pourcentage des fines (\%) & \multicolumn{2}{|c|}{ Fine $(<80 \mu \mathrm{m})$} & \multicolumn{2}{|l|}{63} \\
\hline \multirow{3}{*}{$\begin{array}{l}\text { Distribution de la taille } \\
\text { des grains (\%) }\end{array}$} & \multicolumn{2}{|c|}{ Sable $(>63 \mu \mathrm{m})$} & \multicolumn{2}{|l|}{46} \\
\hline & \multicolumn{2}{|c|}{ Silt (de 2 à $63 \mu \mathrm{m})$} & \multicolumn{2}{|l|}{48} \\
\hline & \multicolumn{2}{|c|}{ Argile $(<2 \mu \mathrm{m})$} & \multicolumn{2}{|l|}{6} \\
\hline Teneur en eau moyenne (\%) & \multicolumn{4}{|l|}{65} \\
\hline \multirow{5}{*}{$\begin{array}{l}\text { Limites d'Atterberg (\%) } \\
\text { VBS (g/100g) }\end{array}$} & \multicolumn{2}{|c|}{ Limite liquidité $W_{L}$} & \multicolumn{2}{|l|}{52.2} \\
\hline & \multicolumn{2}{|c|}{ Limite de plasticité Wp } & \multicolumn{2}{|l|}{42.7} \\
\hline & \multicolumn{2}{|c|}{ Indice de plasticité Ip } & \multicolumn{2}{|l|}{10} \\
\hline & \multicolumn{2}{|c|}{ Indice de consistance Ic } & \multicolumn{2}{|l|}{-1.33} \\
\hline & \multicolumn{2}{|l|}{ VBS } & \multicolumn{2}{|l|}{0.72} \\
\hline Poids spécifique $\gamma_{s}\left(\mathrm{~g} / \mathrm{cm}^{3}\right)$ & \multicolumn{4}{|l|}{2.48} \\
\hline Teneur en matière organique (\%) & \multicolumn{4}{|l|}{12} \\
\hline Teneur en carbone organique (\%) & \multicolumn{4}{|l|}{4} \\
\hline Teneur en carbonate de calcium(\%) & \multicolumn{4}{|l|}{38} \\
\hline \multicolumn{5}{|l|}{ Caractéristiques environnementales } \\
\hline \multirow{7}{*}{ Métaux lourds } & & $\begin{array}{l}\text { Valeur standar } \\
(26 / 04 / 99)\end{array}$ & $\begin{array}{l}\text { Valeur sédiment } \\
\text { (2016) }\end{array}$ & $\begin{array}{l}\text { Valeur sédiment } \\
\text { (2007) }\end{array}$ \\
\hline & $C d(\mu g / g)$ & 4 & 2.25 à 13.75 & 0.2 à 8 \\
\hline & $P b(\mu g / g)$ & 110 & 0.2 à 102.5 & 0.1 à 144 \\
\hline & $\mathrm{Ni}(\mu \mathrm{g} / \mathrm{g})$ & 45 & 3.75 à 43.75 & 0.1 à 15 \\
\hline & $Z n(\mu g / g)$ & 365 & 30.25à 1447 & 19 à 684 \\
\hline & $\mathrm{Cu}(\mu \mathrm{g} / \mathrm{g})$ & 60 & $0.75 a ̀ ~ 892.5$ & 5 à 6039 \\
\hline & $\operatorname{Cr}(\mu g / g)$ & 120 & $<0.05$ à 57 & 3 à 88 \\
\hline Polluants organiques & $7 P C B(\mu g)$ & 1250 & 0.109 à 0.217 & - \\
\hline
\end{tabular}


Mediterranean rocky coasts:

Features, processes, evolution and problems

\subsubsection{Analyse granulométrique}

La distribution granulométrique est déterminée par granulométrie laser sur un nombre important d'échantillons. Les courbes obtenues présentées sur la figure 3a se trouvent dans un fuseau très serré. Ils présentent une granulométrie uniforme $(\mathrm{Cu}>15)$. Il s’agit de limon fin à sableux. La granulométrie est analogue à celle des sédiments de port de pêche avec la dominance de limon et de sable fin et un faible pourcentage d'argile (6\% en moyenne).

\subsubsection{Argilosité}

La figure 3b montre les résultats des mesures des limites d'Atterberg pour les différents sédiments. La position des points se trouve dans le domaine des sols organiques très plastiques avec une consistance très molle (indice de consistance Ic $<0$ ) et un faible potentiel de gonflement $(\mathrm{Ip}<18)$. La valeur de Bleu confirme ce résultat et révèle une faible capacité d’échange cationique (YUKSELEN \& KAYA, 2008).
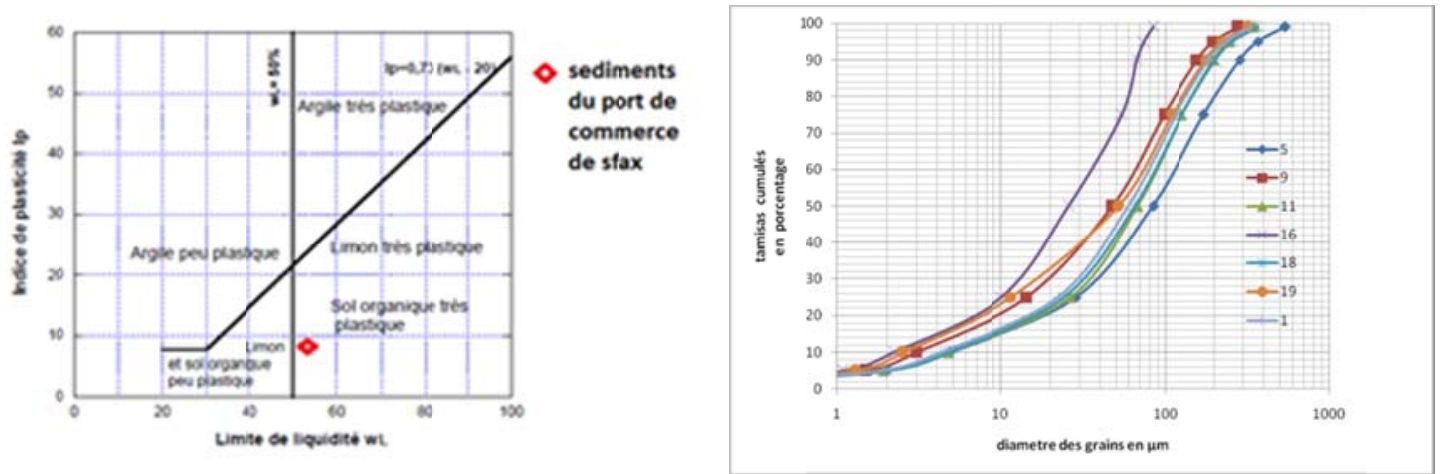

Figure 3. Sédiments du port de commerce de Sfax : Granulométries et diagrammes de plasticité.

\subsection{Caractérisation environnementale}

La teneur en matière organique est déterminée par calcination à $450{ }^{\circ} \mathrm{C}$. Les sédiments du port de commerce de Sfax montrent des teneurs moyennes en matière organique (12 $\%$ en moyenne). Ce pourcentage confère aux sédiments une plasticité moyenne et une capacité à immobiliser les polluants organiques (SCHUMACHER, 2002).

Les analyses des éléments traces contenus dans la matière sèche des sédiments ont été effectuées après une minéralisation des échantillons selon les recommandations NF ISO 11466 (1995). Les résidus dilués sont ensuite analysés par spectrométrie d'absorption atomique.

Les éléments analysés sont respectivement le cadmium $\mathrm{Cd}$, le cuivre $\mathrm{Cu}$, le zinc $\mathrm{Zn}$, le plomb $\mathrm{Pb}$ et le nickel $\mathrm{Ni}$, et le chrome $\mathrm{Cr}$, pour l'ensemble des points d'échantillonnage. Les concentrations obtenues sont ensuite comparées sur le tableau 1 aux seuils d'acceptabilité de rejet en mer (Norme Néerlandaise, in ALZIEU et al, 
Mediterranean rocky coasts:

Features, processes, evolution and problems

1999), aux résultats d'analyses réalisées en 2007 par le BCEOM/SCET-Tunisie (OMMP, 2007). Le choix d'utilisation de la Norme Néerlandaise est du au fait que la Tunisie n'ayant pas établi ses propres normes d'une part, et d'autre part cette classification est l'une des classifications les plus utilisées dans le monde (CJB/EAM, 2007). Les seuils sont largement dépassés pour le cadmium, le cuivre et le zinc. Alors que pour le plomb et le nickel, les concentrations restent à la limite des valeurs seuils, en 2007 et 2016, et elles sont inférieures aux seuils pour le chrome.

La contamination des sédiments par le cadmium est attribuée aux chutes de produits phosphatés lors des opérations d'exportation. Ces teneurs sont accentuées par le panache de pollution transporté par les courants marins depuis la SIAPE jusqu'à le port comme est exposé dans le paragraphe 2. Quant au cuivre la source principale est rattachée à l'unité de peinture localisée à proximité du bassin des voiliers ou sont enregistrées les fortes concentrations. Pour le zinc, les fortes concentrations sont engendrées par la dissolution du zinc à partir des masses pures fixées sur les parties immergées des bateaux pour les protéger contre la corrosion.

Le dépassement des seuils de rejet en mer pour certains éléments traces confirme l’idée de mettre en valeur ces sédiments dans le domaine routier mais sous réserve d'étudier leur comportement vis-à-vis du test de lixiviation.

Pour les polluants organiques (7PCB) contenus dans les sédiments les résultats d'analyses (XP X 33-012, 2000) montrent un non dépassement des seuils autorisés.

\subsection{Caractérisation minéralogique}

La caractérisation minéralogique est indispensable pour toute étude de valorisation ultérieure. Les phases minéralogiques présentes dans les sédiments sont identifiées par diffraction des rayons $\mathrm{X}$ au moyen d'un diffractomètre aux Rayons $\mathrm{X}$ de système XPERT-PRO. Les résultats d'analyses sont ensuite traités avec le logiciel X'pert HighScoredonner pour estimer les pourcentages des différents composés chimiques présents dans les sédiments.

\subsubsection{Analyse des diffractogrammes}

Les diffractogrammes obtenus pour les différents échantillons sont assez similaires. Sur la figure 4 est présenté le diffractogramme de l'échantillon 5, calciné et broyé à $450{ }^{\circ} \mathrm{C}$. Le dépouillement du diffractogramme montre la dominance du quartz $\left(\mathrm{SiO}_{2}\right)$ justifié par le fort pourcentage de sable et de limon, la présence des carbonates de calcium sous ces deux formes (calcite et dolomite), de l'anhydrite produit par calcination du gypse et de l'halite en pourcentage notable. 
Mediterranean rocky coasts:

Features, processes, evolution and problems

\subsubsection{Composition minéralogique des sédiments}

Une estimation semi quantitative $( \pm 5 \%)$ des différents composés chimiques présents dans les sédiments est réalisée à partir des diffractogrammes et au moyen du logiciel X'pert HighScoreet. Les résultats obtenus sont présentés sur le tableau 2.

La répartition massique des composés chimiques dans les sédiments du port de commerce de Sfax montre qu'ils formés essentiellement de quartz et de carbonates de calcium et en pourcentages moindres du gypse et de halite.

Ces fortes teneurs en sulfates expliquent la surestimation du COT déduit par la différence de poids après calcination. En effet la transformation du gypse en anhydrite se fait par libération de la vapeur d'eau. Les sulfates proviennent essentiellement des phosphates et du soufre importé quant aux cristaux de sel ils proviennent de la saline qui longe la côte de Sfax.

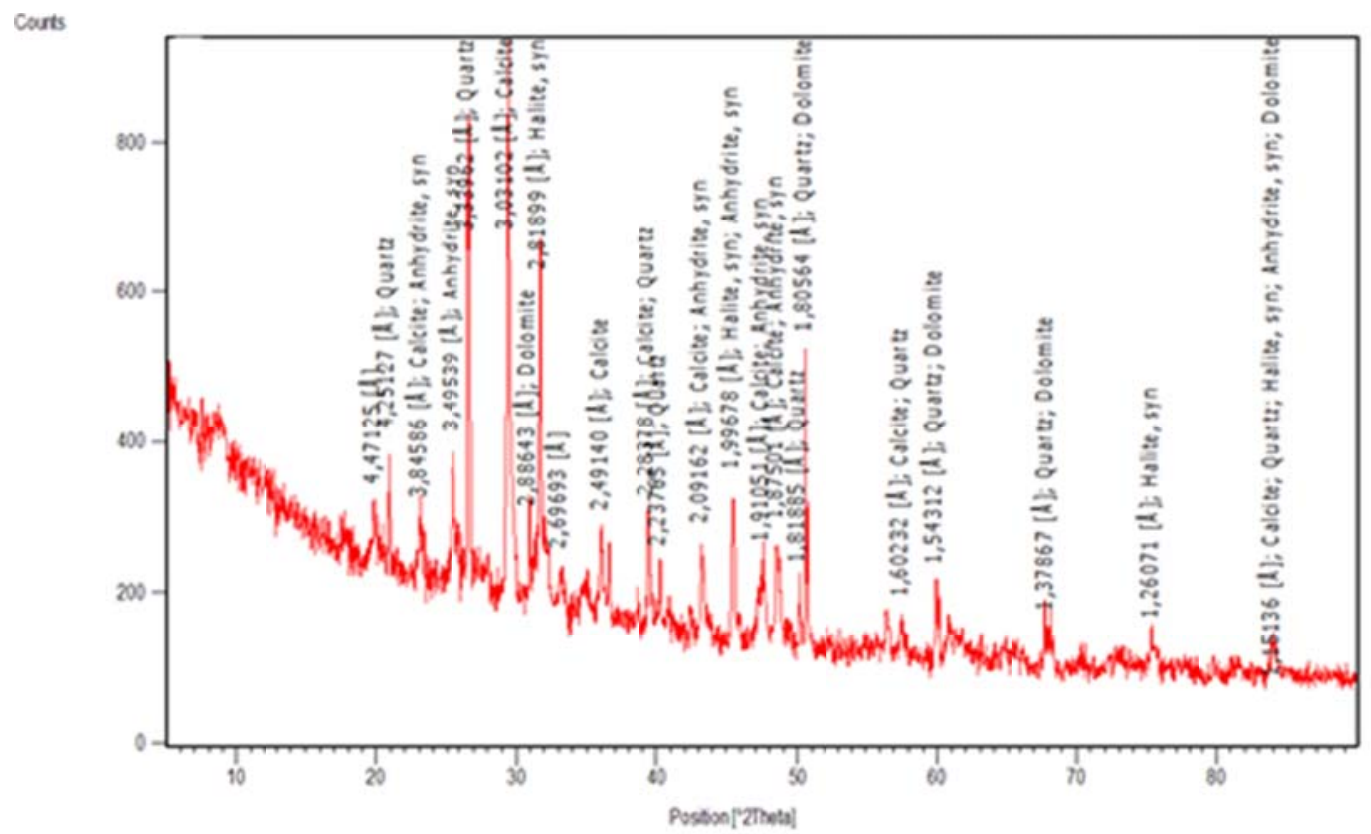

Figure 4. Diffractogramme des sédiments du port de commerce de Sfax.

Tableau 2. Composition minéralogique des sédiments de port de commerce.

\begin{tabular}{|l|l|l|}
\hline & Composé chimique & Pourcentage \\
\hline Quartz & $\mathrm{SiO}_{2}$ & 35.86 \\
\hline Calcite & $\mathrm{CaCO}_{3}$ & 35.88 \\
\hline Dolomite & $\mathrm{CaMg}\left(\mathrm{CO}_{3}\right) 2$ & 2.8 \\
\hline Gypse & $\left.(\mathrm{CaSO})_{4}\right)_{2} \mathrm{O}$ & 11 \\
\hline Halite & $\mathrm{NaCl}$ & 14.44 \\
\hline
\end{tabular}

\section{Conclusion}

Les essais de caractérisation physique des sédiments du port de commerce de Sfax ont montré des propriétés similaires à celles des sédiments du port de pêche de Sfax. La 
Mediterranean rocky coasts:

Features, processes, evolution and problems

distribution granulométrique et les forts pourcentages en matière organique (supérieur à $3 \%$ ) leurs confèrent le comportement de sols organiques très plastiques de faibles potentiels de gonflement. Selon la classification GTR, ils se positionnent dans la classe F.

Les fortes teneurs en sels nécessitent un traitement préalable (dé-salement par exemple) avant toute valorisation ultérieure surtout comme matériau de construction routière.

L'analyse environnementale a confirmé les résultats des analyses précédentes et qui datent de 2007. Les concentrations dépassent les seuils de rejet en mer notamment pour le cuivre, le cadmium et le zinc. Pour une meilleure préservation de l'environnement marin il est devenu indispensable de développer des procédures de valorisation de ces sédiments notamment dans une application routière. Des essais de lixiviation sont nécessaires pour vérifier la mobilité de ces éléments à différentes valeurs de $\mathrm{pH}$.

\section{Références bibliographiques}

ALZIEU C., ABARNOU A., BASSOULLET P., BOUTIER B. (1999). Dragage et environnement marin : état des connaissances. Edition Ifremer, France, 209 p.

CJB/EAM. (2007). Etude d'impact sur l'environnement du dragage du complexe portuaire La Goulette-Radès. Office de la Marine Marchande et des ports, CJB Environnement Inc., Environmental Assessment and Management, 109 p.

COMETE ENGINEERING. (2008). Etude de dépollution et de réhabilitation des côtes sud de Sfax. Rapport de synthèse. Comete Engineering code 26079, 36 p.

FEKI N., MBARKA M. (2015). Caractérisation physique et environnementale des sédiments du port de pêche de Sfax. CM2, Ferrare, Italie, pp. 137-142. doi:10.5150/cmcm.2015.028

GTR. (2000). Guide technique pour la réalisation des remblais et des couches de forme. Fascicules I \& II, Editions du SETRA-LCPC, France.

NF ISO 11466. (1995). Qualité du sol - Extraction des éléments en traces solubles dans l'eau régale. AFNOR Editions, France.

OMMP. (2007). Rapport annuel de l'Office de la Marine Marchande et des Ports, Tunisie, 46p.

SCHUMACHER B. A. (2002). Methods for the determination of total organic carbon (TOC) in soils and sediments. United States Environmental Protection Agency, Environmental Sciences, Division National, Las Vegas, NCEA-C- 1282 EMASC-001, Report EPA/600/R-02/069, 25 p.

XP X33-012. (2000). Caractérisation des boues. Dosage des hydrocarbures aromatiques polycycliques (HAP) et des polychlorobiphényles (PCB). AFNOR Editions, France.

YUKSELEN Y., KAYA A. (2008). Suitability of the methylene blue test for surface area, cation exchange capacity and swell potential determination of clayey soils. Engineering Geology, Vol. 102, pp. 38 - 45. https://doi.org/10.1016/j.enggeo.2008.07.002 